\title{
Weighted Composition Operators from Weighted Bergman Spaces to Weighted-Type Spaces on the Upper Half-Plane
}

\author{
Stevo Stević, ${ }^{1}$ Ajay K. Sharma, ${ }^{2}$ and S. D. Sharma ${ }^{3}$ \\ ${ }^{1}$ Mathematical Institute of the Serbian Academy of Sciences, Knez Mihailova 36/III, 11000 Beograd, Serbia \\ ${ }^{2}$ School of Mathematics, Shri Mata Vaishno Devi University, Kakryal, J \& K Katra 182320, India \\ ${ }^{3}$ Department of Mathematics, University of Jammu, Jammu 180006, India \\ Correspondence should be addressed to Stevo Stević, sstevic@ptt.rs \\ Received 25 January 2011; Accepted 3 May 2011 \\ Academic Editor: Narcisa C. Apreutesei \\ Copyright (C) 2011 Stevo Stević et al. This is an open access article distributed under the Creative \\ Commons Attribution License, which permits unrestricted use, distribution, and reproduction in \\ any medium, provided the original work is properly cited. \\ Let $\psi$ be a holomorphic mapping on the upper half-plane $\Pi^{+}=\{z \in \mathbb{C}: \mathfrak{J} z>0\}$ and $\varphi$ be \\ a holomorphic self-map of $\Pi^{+}$. We characterize bounded weighted composition operators acting \\ from the weighted Bergman space to the weighted-type space on the upper half-plane. Under a \\ mild condition on $\psi$, we also characterize the compactness of these operators.
}

\section{Introduction and Preliminaries}

Let $\Pi^{+}=\{z \in \mathbb{C}: \Im z>0\}$ be the upper half-plane, $\Omega$ a domain in $\mathbb{C}$ or $\mathbb{C}^{n}$, and $H(\Omega)$ the space of all holomorphic functions on $\Omega$. Let $\psi \in H(\Omega)$, and let $\varphi$ be a holomorphic self-map of $\Omega$. Then by

$$
W_{\varphi, \psi}(f)(z)=\psi(f \circ \varphi)(z), \quad z \in \Omega,
$$

is defined a linear operator on $H(\Omega)$ which is called weighted composition operator. If $\psi(z)=$ 1 , then $W_{\varphi, \psi}$ becomes composition operator and is denoted by $C_{\varphi}$, and if $\varphi(z)=z$, then $W_{\varphi, \psi}$ becomes multiplication operator and is denoted by $M_{\psi}$.

During the past few decades, composition operators and weighted composition operators have been studied extensively on spaces of holomorphic functions on various domains in $\mathbb{C}$ or $\mathbb{C}^{n}$ (see, e.g., [1-22] and the references therein). There are many reasons for this interest, for example, it is well known that the surjective isometries of Hardy and 
Bergman spaces are certain weighted composition operators (see [23, 24]). For some other operators related to weighted composition operators, see [25-30] and the references therein.

While there is a vast literature on composition and weighted composition operators between spaces of holomorphic functions on the unit disk $\mathbb{D}$, there are few papers on these and related operators on spaces of functions holomorphic in the upper half-plane (see, e.g., $[2,3,5,7-9,11,12,16-18,31]$ and the references therein). For related results in the setting of the complex plane see also papers [19-21].

The behaviour of composition operators on spaces of functions holomorphic in the upper half-plane is considerably different from the behaviour of composition operators on spaces of functions holomorphic in the unit disk $\mathbb{D}$. For example, there are holomorphic selfmaps of $\Pi^{+}$which do not induce composition operators on Hardy and Bergman spaces on the upper half-plane, whereas it is a well-known consequence of the Littlewood subordination principle that every holomorphic self-map $\varphi$ of $\mathbb{D}$ induces a bounded composition operator on the Hardy and weighted Bergman spaces on $\mathbb{D}$. Also, Hardy and Bergman spaces on the upper half-plane do not support compact composition operators (see $[3,5])$.

For $0<p<\infty$ and $\alpha \in(-1, \infty)$, let $\mathfrak{L}^{p}\left(\Pi^{+}, d A_{\alpha}\right)$ denote the collection of all Lebesgue $p$-integrable functions $f: \Pi^{+} \rightarrow \mathbb{C}$ such that

$$
\int_{\Pi^{+}}|f(z)|^{p} d A_{\alpha}(z)<\infty
$$

where

$$
d A_{\alpha}(z)=\frac{1}{\pi}(\alpha+1)(2 \mathfrak{I} z)^{\alpha} d A(z)
$$

$d A(z)=d x d y$, and $z=x+i y$.

Let $\mathcal{A}_{\alpha}^{p}\left(\Pi^{+}\right)=\mathfrak{L}^{p}\left(\Pi^{+}, d A_{\alpha}\right) \cap H\left(\Pi^{+}\right)$. For $1 \leq p<\infty, \mathcal{A}_{\alpha}^{p}\left(\Pi^{+}\right)$is a Banach space with the norm defined by

$$
\|f\|_{A_{\alpha}^{p}\left(\Pi^{+}\right)}=\left(\int_{\Pi^{+}}|f(z)|^{p} d A_{\alpha}(z)\right)^{1 / p}<\infty
$$

With this norm $\mathcal{A}_{\alpha}^{p}\left(\Pi^{+}\right)$becomes a Banach space when $p \geq 1$, while for $p \in(0,1)$ it is a Fréchet space with the translation invariant metric

$$
d(f, g)=\|f-g\|_{\mathcal{A}_{\alpha}^{p}\left(\Pi^{+}\right)^{\prime}}^{p} \quad f, g \in \mathscr{A}_{\alpha}^{p}\left(\Pi^{+}\right)
$$

Recall that for every $f \in \mathcal{A}_{\alpha}^{p}\left(\Pi^{+}\right)$the following estimate holds:

$$
|f(x+i y)|^{p} \leq C \frac{\|f\|_{A_{\alpha}^{p}\left(\Pi^{+}\right)}^{p}}{y^{\alpha+2}},
$$

where $C$ is a positive constant independent of $f$. 
Let $\beta>0$. The weighted-type space (or growth space) on the upper half-plane $\mathcal{A}_{\beta}^{\infty}\left(\Pi^{+}\right)$ consists of all $f \in H\left(\Pi^{+}\right)$such that

$$
\|f\|_{A_{\beta}^{\infty}\left(\Pi^{+}\right)}=\sup _{z \in \Pi^{+}}(\Im z)^{\beta}|f(z)|<\infty
$$

It is easy to check that $\mathscr{A}_{\beta}^{\infty}\left(\Pi^{+}\right)$is a Banach space with the norm defined above. For weightedtype spaces on the unit disk, polydisk, or the unit ball see, for example, papers $[10,32,33]$ and the references therein.

Given two Banach spaces $Y$ and $Z$, we recall that a linear map $T: Y \rightarrow Z$ is bounded if $T(E) \subset Z$ is bounded for every bounded subset $E$ of $Y$. In addition, we say that $T$ is compact if $T(E) \subset Z$ is relatively compact for every bounded set $E \subset Y$.

In this paper, we consider the boundedness and compactness of weighted composition operators acting from $\mathcal{A}_{\alpha}^{p}\left(\Pi^{+}\right)$to the weighted-type space $\mathcal{A}_{\beta}^{\infty}\left(\Pi^{+}\right)$. Related results on the unit disk and the unit ball can be found, for example, in $[6,13,15]$.

Throughout this paper, constants are denoted by $C$; they are positive and may differ from one occurrence to the other. The notation $a \leq b$ means that there is a positive constant $C$ such that $a \leq C b$. Moreover, if both $a \leq b$ and $b \leq a$ hold, then one says that $a \asymp b$.

\section{Main Results}

The boundedness and compactness of the weighted composition operator $W_{\varphi, \psi}: \mathscr{A}_{\alpha}^{p}\left(\Pi^{+}\right) \rightarrow$ $\mathcal{A}_{\beta}^{\infty}\left(\Pi^{+}\right)$are characterized in this section.

Theorem 2.1. Let $1 \leq p<\infty, \alpha>-1, \beta>0, \psi \in H\left(\Pi^{+}\right)$, and let $\varphi$ be a holomorphic self-map of $\Pi^{+}$. Then $W_{\varphi, \psi}: \mathcal{A}_{\alpha}^{p}\left(\Pi^{+}\right) \rightarrow \mathcal{A}_{\beta}^{\infty}\left(\Pi^{+}\right)$is bounded if and only if

$$
M:=\sup _{z \in \Pi^{+}} \frac{(\Im z)^{\beta}}{(\Im \varphi(z))^{(\alpha+2) / p}}|\psi(z)|<\infty .
$$

Moreover, if the operator $W_{\varphi, \psi}: \mathcal{A}_{\alpha}^{p}\left(\Pi^{+}\right) \rightarrow \mathcal{A}_{\beta}^{\infty}\left(\Pi^{+}\right)$is bounded then the following asymptotic relationship holds:

$$
\left\|W_{\varphi, \psi}\right\|_{\mathcal{A}_{\alpha}^{p}\left(\Pi^{+}\right) \rightarrow A_{\beta}^{\infty}\left(\Pi^{+}\right)} \asymp M .
$$

Proof. First suppose that (2.1) holds. Then for any $z \in \Pi^{+}$and $f \in \mathcal{A}_{\alpha}^{p}\left(\Pi^{+}\right)$, by (1.6) we have

$$
(\Im z)^{\beta}\left|\left(W_{\varphi, \psi} f\right)(z)\right|=(\Im z)^{\beta}|\psi(z)||f(\varphi(z))| \leq \frac{(\Im z)^{\beta}}{(\Im \varphi(z))^{(\alpha+2) / p}}|\psi(z)|\|f\|_{\mathscr{A}_{\alpha}^{p}\left(\Pi^{+}\right)^{\prime}}
$$

and so by (2.1), $W_{\varphi, \psi}: \mathcal{A}_{\alpha}^{p}\left(\Pi^{+}\right) \rightarrow \mathcal{A}_{\beta}^{\infty}\left(\Pi^{+}\right)$is bounded and moreover

$$
\left\|W_{\varphi, \psi}\right\|_{A_{\alpha}^{p}\left(\Pi^{+}\right) \rightarrow A_{\beta}^{\infty}\left(\Pi^{+}\right)} \preceq M .
$$


Conversely suppose $W_{\varphi, \psi}: \mathcal{A}_{\alpha}^{p}\left(\Pi^{+}\right) \rightarrow \mathcal{A}_{\beta}^{\infty}\left(\Pi^{+}\right)$is bounded. Consider the function

$$
f_{w}(z)=\frac{(\Im w)^{(\alpha+2) / p}}{(z-\bar{w})^{(2 \alpha+4) / p}}, \quad w \in \Pi^{+} .
$$

Then $f_{w} \in \mathcal{A}_{\alpha}^{p}\left(\Pi^{+}\right)$and moreover $\sup _{w \in \Pi^{+}}\left\|f_{w}\right\|_{\mathcal{A}_{\alpha}^{p}\left(\Pi^{+}\right)} \preceq 1$ (see, e.g., Lemma 1 in [18]).

Thus the boundedness of $W_{\varphi, \psi}: \mathcal{A}_{\alpha}^{p}\left(\Pi^{+}\right) \rightarrow \mathcal{A}_{\beta}^{\infty}\left(\Pi^{+}\right)$implies that

$$
(\Im z)^{\beta}|\psi(z)|\left|f_{w}(\varphi(z))\right| \leq\left\|W_{\varphi, \psi} f_{w}\right\|_{\mathcal{A}_{\beta}^{\infty}\left(\Pi^{+}\right)} \preccurlyeq\left\|W_{\varphi, \psi}\right\|_{\mathcal{A}_{\alpha}^{p}\left(\Pi^{+}\right) \rightarrow \mathcal{A}_{\beta}^{\infty}\left(\Pi^{+}\right)}
$$

for every $z, w \in \Pi^{+}$. In particular, if $z \in \Pi^{+}$is fixed then for $w=\varphi(z)$, we get

$$
\frac{(\Im z)^{\beta}}{(\Im \varphi(z))^{(\alpha+2) / p}}|\psi(z)| \leq\left\|W_{\varphi, \psi}\right\|_{\mathcal{A}_{\alpha}^{p}\left(\Pi^{+}\right) \rightarrow A_{\beta}^{\infty}\left(\Pi^{+}\right)} .
$$

Since $z \in \Pi^{+}$is arbitrary, (2.1) follows and moreover

$$
M \preceq\left\|W_{\varphi, \psi}\right\|_{\mathcal{A}_{\alpha}^{p}\left(\Pi^{+}\right) \rightarrow A_{\beta}^{\infty}\left(\Pi^{+}\right)} .
$$

If $W_{\varphi, \psi}: \mathscr{A}_{\alpha}^{p}\left(\Pi^{+}\right) \rightarrow \mathcal{A}_{\beta}^{\infty}\left(\Pi^{+}\right)$is bounded then from (2.4) and (2.8) asymptotic relationship (2.2) follows.

Corollary 2.2. Let $1 \leq p<\infty, \alpha>-1$, and $\beta>0$ be such that $\beta p \geq \alpha+2$ and $\psi \in H\left(\Pi^{+}\right)$. Then $M_{\psi}: \mathcal{A}_{\alpha}^{p}\left(\Pi^{+}\right) \rightarrow \mathcal{A}_{\beta}^{\infty}\left(\Pi^{+}\right)$is bounded if and only if $\psi \in X$, where

$$
X= \begin{cases}\mathcal{A}_{\beta-((\alpha+2) / p)}^{\infty}\left(\Pi^{+}\right) & \text {if } \alpha+2<\beta p, \\ H^{\infty}\left(\Pi^{+}\right) & \text {if } \alpha+2=\beta p .\end{cases}
$$

Example 2.3. Let $1 \leq p<\infty, \alpha>-1$ and $\beta>0$ be such that $\beta p \geq \alpha+2$ and $w \in \Pi^{+}$. Let $\psi_{w}$ be a holomorphic map of $\Pi^{+}$defined as

$$
\psi_{w}(z)= \begin{cases}\frac{1}{(z-\bar{w})^{\beta-((\alpha+2) / p)}} & \text { if } \alpha+2<\beta p \\ \frac{\Im w}{z-\bar{w}} & \text { if } \alpha+2=\beta p .\end{cases}
$$


For $z=x+i y$ and $w=u+i v$ in $\Pi^{+}$, we have

$$
\begin{aligned}
\sup _{z \in \Pi^{+}}(\Im z)^{\beta-(\alpha+2) / p}\left|\psi_{w}(z)\right| & =\sup _{z=x+i y \in \Pi^{+}} \frac{y^{\beta-(\alpha+2) / p}}{\left((x-u)^{2}+(y+v)^{2}\right)^{(\beta p-(\alpha+2)) / 2 p}} \\
& \leq \sup _{z=x+i y \in \Pi^{+}} \frac{y^{\beta-(\alpha+2) / p}}{(y+v)^{\beta-(\alpha+2) / p}} \leq 1 .
\end{aligned}
$$

Thus $\psi_{w} \in \mathcal{A}_{\beta-(\alpha+2) / p}^{\infty}\left(\Pi^{+}\right)$if $\alpha+2<\beta p$. Similarly $\psi_{w} \in H^{\infty}\left(\Pi^{+}\right)$if $\alpha+2=\beta p$. By Corollary 2.2, it follows that $M_{\psi_{w}}: \mathcal{A}_{\alpha}^{p}\left(\Pi^{+}\right) \rightarrow \mathcal{A}_{\beta}^{\infty}\left(\Pi^{+}\right)$is bounded.

Corollary 2.4. Let $1 \leq p<\infty, \alpha>-1, \beta>0$, and let $\varphi$ be a holomorphic self-map of $\Pi^{+}$. Then $C_{\varphi}: \mathcal{A}_{\alpha}^{p}\left(\Pi^{+}\right) \rightarrow \mathcal{A}_{\beta}^{\infty}\left(\Pi^{+}\right)$is bounded if and only if

$$
\sup _{z \in \Pi^{+}} \frac{(\Im z)^{\beta}}{(\Im \varphi(z))^{(\alpha+2) / p}}<\infty .
$$

Corollary 2.5. Let $\varphi$ be the linear fractional map

$$
\varphi(z)=\frac{a z+b}{c z+d}, \quad a, b, c, d \in \mathbb{R}, a d-b c>0 .
$$

Then necessary and sufficient condition that $C_{\varphi}: \mathcal{A}_{\alpha}^{p}\left(\Pi^{+}\right) \rightarrow \mathcal{A}_{\beta}^{\infty}\left(\Pi^{+}\right)$is bounded is that $c=0$ and $\alpha+2=\beta p$.

Proof. Assume that $C_{\varphi}: \mathcal{A}_{\alpha}^{p}\left(\Pi^{+}\right) \rightarrow \mathcal{A}_{\beta}^{\infty}\left(\Pi^{+}\right)$is bounded. Then

$$
\sup _{z \in \Pi^{+}} \frac{(\Im z)^{\beta}}{(\Im \varphi(z))^{(\alpha+2) / p}}=\sup _{z=x+i y \in \Pi^{+}} \frac{\left((c x+d)^{2}+c^{2} y^{2}\right)^{(\alpha+2) / p} y^{\beta}}{(a d-b c)^{(\alpha+2) / p} y^{(\alpha+2) / p}}
$$

which is finite only if $c=0$ and $\alpha+2=\beta p$.

Conversely, if $c=0$ and $\alpha+2=\beta p$, then from (2.13) we get $a \neq 0$, and by some calculation

$$
\sup _{z \in \Pi^{+}} \frac{(\Im \mathfrak{I} z)^{\beta}}{(\Im \varphi(z))^{(\alpha+2) / p}}=\left(\frac{d}{a}\right)^{\beta}<\infty .
$$

Hence $C_{\varphi}: \mathcal{A}_{\alpha}^{p}\left(\Pi^{+}\right) \rightarrow \mathcal{A}_{\beta}^{\infty}\left(\Pi^{+}\right)$is bounded.

Corollary 2.6. Let $1 \leq p<\infty, \alpha>-1$, and $\beta>0$ be such that $\beta p=\alpha+2$. Let $\varphi$ be a holomorphic self-map of $\Pi^{+}$and $\psi=\left(\varphi^{\prime}\right)^{\beta}$. Then the weighted composition operator $W_{\varphi, \psi}$ acts boundedly from $\mathcal{A}_{\alpha}^{p}\left(\Pi^{+}\right)$to $\mathscr{A}_{\beta}^{\infty}\left(\Pi^{+}\right)$. 
Proof. By Theorem 2.1, $W_{\varphi, \psi}: \mathcal{A}_{\alpha}^{p}\left(\Pi^{+}\right) \rightarrow \mathcal{A}_{\beta}^{\infty}\left(\Pi^{+}\right)$is bounded if and only if

$$
\sup _{z \in \Pi^{+}} \frac{(\Im z)^{\beta}}{(\Im \varphi(z))^{\beta}}\left|\varphi^{\prime}(z)\right|^{\beta}<\infty .
$$

By the Schwarz-Pick theorem on the upper half-plane we have that for every holomorphic self-map $\varphi$ of $\Pi^{+}$and all $z \in \Pi^{+}$

$$
\frac{\left|\varphi^{\prime}(z)\right|}{\Im \varphi(z)} \leq \frac{1}{\Im z}
$$

where the equality holds when $\varphi$ is a Möbius transformation given by (2.13). From (2.17), condition (2.16) follows and consequently the boundedness of the operator $W_{\varphi, \psi}: \mathcal{A}_{\alpha}^{p}\left(\Pi^{+}\right) \rightarrow$ $\mathcal{A}_{\beta}^{\infty}\left(\Pi^{+}\right)$.

Corollary 2.6 enables us to show that there exist $1 \leq p<\infty, \alpha>-1, \beta>0$, and holomorphic maps $\varphi$ and $\psi$ of the upper half-plane $\Pi^{+}$such that neither $C_{\varphi}: \mathcal{A}_{\alpha}^{p}\left(\Pi^{+}\right) \rightarrow$ $\mathscr{A}_{\beta}^{\infty}\left(\Pi^{+}\right)$nor $M_{\psi}: \mathcal{A}_{\alpha}^{p}\left(\Pi^{+}\right) \rightarrow \mathcal{A}_{\beta}^{\infty}\left(\Pi^{+}\right)$is bounded, but $W_{\varphi, \psi}: \mathcal{A}_{\alpha}^{p}\left(\Pi^{+}\right) \rightarrow \mathcal{A}_{\beta}^{\infty}\left(\Pi^{+}\right)$is bounded.

Example 2.7. Let $1 \leq p<\infty, \alpha>-1$, and $\beta>0$ be such that $\beta p=\alpha+2$. Let $\varphi(z)=(a z+b) /(c z+$ d), $a, b, c, d \in \mathbb{R}, a d-b c>0$, and $c \neq 0$. Then by Corollary 2.5, $C_{\varphi}: \mathcal{A}_{\alpha}^{p}\left(\Pi^{+}\right) \rightarrow \mathcal{A}_{\beta}^{\infty}\left(\Pi^{+}\right)$is not bounded. On the other hand, if

$$
\psi(z)=\left(\varphi^{\prime}(z)\right)^{\beta}=\left(\frac{a d-b c}{(c z+d)^{2}}\right)^{\beta},
$$

then $\psi \notin H^{\infty}\left(\Pi^{+}\right)$and so by Corollary 2.2, $M_{\psi}: \mathcal{A}_{\alpha}^{p}\left(\Pi^{+}\right) \rightarrow \mathcal{A}_{\beta}^{\infty}\left(\Pi^{+}\right)$is not bounded. However, by Corollary 2.6, we have that $W_{\varphi,\left(\varphi^{\prime}\right)^{\beta}}: \mathcal{A}_{\alpha}^{p}\left(\Pi^{+}\right) \rightarrow \mathcal{A}_{\beta}^{\infty}\left(\Pi^{+}\right)$is bounded.

The next Schwartz-type lemma characterizes compact weighted composition operators $W_{\varphi, \psi}: \mathcal{A}_{\alpha}^{p}\left(\Pi^{+}\right) \rightarrow \mathcal{A}_{\beta}^{\infty}\left(\Pi^{+}\right)$and it follows from standard arguments ([4]).

Lemma 2.8. Let $1 \leq p<\infty, \alpha>-1, \beta>0, \psi \in H\left(\Pi^{+}\right)$, and let $\varphi$ be a holomorphic self-map of $\Pi^{+}$. Then $W_{\varphi, \psi}: \mathcal{A}_{\alpha}^{p}\left(\Pi^{+}\right) \rightarrow \mathcal{A}_{\beta}^{\infty}\left(\Pi^{+}\right)$is compact if and only if, for any bounded sequence $\left(f_{n}\right)_{n \in \mathbb{N}} \subset$ $\mathcal{A}_{\alpha}^{p}\left(\Pi^{+}\right)$converging to zero on compacts of $\Pi^{+}$, one has

$$
\lim _{n \rightarrow \infty}\left\|W_{\varphi, \psi} f_{n}\right\|_{\mathscr{A}_{\beta}^{\infty}\left(\Pi^{+}\right)}=0 .
$$

Theorem 2.9. Let $1 \leq p<\infty, \alpha>-1, \beta>0, \psi \in H\left(\Pi^{+}\right)$and $\varphi$ be a holomorphic self-map of $\Pi^{+}$. If $W_{\varphi, \psi}: \mathcal{A}_{\alpha}^{p}\left(\Pi^{+}\right) \rightarrow \mathcal{A}_{\beta}^{\infty}\left(\Pi^{+}\right)$is compact, then

$$
\lim _{r \rightarrow 0} \sup _{\Im \varphi(z)<r} \frac{(\Im z)^{\beta}}{(\Im \varphi(z))^{(\alpha+2) / p}}|\psi(z)|=0 .
$$


Proof. Suppose $W_{\varphi, \psi}: \mathscr{A}_{\alpha}^{p}\left(\Pi^{+}\right) \rightarrow \mathcal{A}_{\beta}^{\infty}\left(\Pi^{+}\right)$is compact and (2.20) does not hold. Then there is a $\delta>0$ and a sequence $\left(z_{n}\right)_{n \in \mathbb{N}} \subset \Pi^{+}$such that $\Im \varphi\left(z_{n}\right) \rightarrow 0$ and

$$
\frac{\left(\Im z_{n}\right)^{\beta}}{\left(\Im \varphi\left(z_{n}\right)\right)^{(\alpha+2) / p}}\left|\psi\left(z_{n}\right)\right|>\delta
$$

for all $n \in \mathbb{N}$. Let $w_{n}=\varphi\left(z_{n}\right), n \in \mathbb{N}$, and

$$
f_{n}(z)=\frac{\left(\Im w_{n}\right)^{(\alpha+2) / p}}{\left(z-\bar{w}_{n}\right)^{(2 \alpha+4) / p}}, \quad n \in \mathbb{N} .
$$

Then $f_{n}$ is a norm bounded sequence and $f_{n} \rightarrow 0$ on compacts of $\Pi^{+}$as $\Im \varphi\left(z_{n}\right) \rightarrow 0$. By Lemma 2.8 it follows that

$$
\lim _{n \rightarrow \infty}\left\|W_{\varphi, \psi} f_{n}\right\|_{\mathcal{A}_{\beta}^{\infty}\left(\Pi^{+}\right)}=0
$$

On the other hand,

$$
\begin{aligned}
\left\|W_{\varphi, \psi} f_{n}\right\|_{\mathcal{A}_{\beta}^{\infty}\left(\Pi^{+}\right)} & \geq\left(\Im z_{n}\right)^{\beta}\left|\left(W_{\varphi, \psi} f_{n}\right)\left(z_{n}\right)\right| \\
& =\left(\mathfrak{I} z_{n}\right)^{\beta}\left|\psi\left(z_{n}\right)\right|\left|f_{n}\left(\varphi\left(z_{n}\right)\right)\right| \\
& =\frac{\left(\Im z_{n}\right)^{\beta}}{2^{(2 \alpha+4) / p}\left(\Im \varphi\left(z_{n}\right)\right)^{(\alpha+2) / p}}\left|\psi\left(z_{n}\right)\right|>\frac{\delta}{2^{(2 \alpha+4) / p}},
\end{aligned}
$$

which is a contradiction. Hence (2.20) must hold, as claimed.

Before we formulate and prove a converse of Theorem 2.9, we define, for every $a, b \in$ $(0, \infty)$ such that $a<b$, the following subset of $\Pi^{+}$:

$$
\Gamma_{a, b}=\left\{z \in \Pi^{+}: a \leq \Im z \leq b\right\}
$$

Theorem 2.10. Let $1 \leq p<\infty, \alpha>-1, \beta>0, \psi \in H\left(\Pi^{+}\right)$, and let $\varphi$ be a holomorphic self-map of $\Pi^{+}$and $W_{\varphi, \psi}: \mathcal{A}_{\alpha}^{p}\left(\Pi^{+}\right) \rightarrow \mathcal{A}_{\beta}^{\infty}\left(\Pi^{+}\right)$be bounded. Suppose that $\psi \in \mathcal{A}_{\beta}^{\infty}\left(\Pi^{+}\right)$and $(\mathfrak{I} z)^{\beta}|\psi(z)| \rightarrow 0$ as $|\Re \varphi(z)| \rightarrow \infty$ within $\Gamma_{a, b}$ for all $a$ and $b, 0<a<b<\infty$. Then $W_{\varphi, \psi}: \mathcal{A}_{\alpha}^{p}\left(\Pi^{+}\right) \rightarrow \mathcal{A}_{\beta}^{\infty}\left(\Pi^{+}\right)$is compact if condition (2.20) holds.

Proof. Assume (2.20) holds. Then for each $\varepsilon>0$, there is an $M_{1}>0$ such that

$$
\frac{(\Im z)^{\beta}}{(\Im \varphi(z))^{(\alpha+2) / p}}|\psi(z)|<\varepsilon, \quad \text { whenever } \Im \varphi(z)<M_{1} .
$$


Let $\left(f_{n}\right)_{n \in \mathbb{N}}$ be a sequence in $\mathcal{A}_{\alpha}^{p}\left(\Pi^{+}\right)$such that $\sup _{n \in \mathbb{N}}\left\|f_{n}\right\|_{\mathcal{A}_{\alpha}^{p}\left(\Pi^{+}\right)} \leq M$ and $f_{n} \rightarrow 0$ uniformly on compact subsets of $\Pi^{+}$as $n \rightarrow \infty$. Thus for $z \in \Pi^{+}$such that $\Im \varphi(z)<M_{1}$ and each $n \in \mathbb{N}$, we have

$$
(\Im z)^{\beta}|\psi(z)|\left|f_{n}(\varphi(z))\right| \leq \frac{(\Im z)^{\beta}}{(\Im \varphi(z))^{(\alpha+2) / p}}|\psi(z)|\left\|f_{n}\right\|_{\AA_{\alpha}^{p}\left(\Pi^{+}\right)}<\varepsilon M .
$$

From estimate (1.6) we have

$$
\left|f_{n}(z)\right| \preceq \frac{\left\|f_{n}\right\|_{\mathcal{A}_{\alpha}^{p}\left(\Pi^{+}\right)}}{(\Im z)^{(\alpha+2) / p}} \preceq \frac{M}{(\Im z)^{(\alpha+2) / p}} .
$$

Thus there is an $M_{2}>M_{1}$ such that

$$
\left|f_{n}(\varphi(z))\right|<\varepsilon,
$$

whenever $\Im \varphi(z)>M_{2}$. Hence for $z \in \Pi^{+}$such that $\Im \varphi(z)>M_{2}$ and each $n \in \mathbb{N}$ we have

$$
(\Im z)^{\beta}|\psi(z)|\left|f_{n}(\varphi(z))\right|<\varepsilon\|\psi\|_{\mathcal{A}_{\beta}^{\infty}\left(\Pi^{+}\right)} .
$$

If $M_{1} \leq \Im \varphi(z) \leq M_{2}$, then by the assumption there is an $M_{3}>0$ such that $(\Im z)^{\beta}|\psi(z)|<\varepsilon$, whenever $|\Re \varphi(z)|>M_{3}$. Therefore, for each $n \in \mathbb{N}$ we have

$$
(\Im z)^{\beta}|\psi(z)|\left|f_{n}(\varphi(z))\right| \leq \varepsilon \frac{\left\|f_{n}\right\|_{\mathscr{A}_{\alpha}^{p}\left(\Pi^{+}\right)}}{(\Im \varphi(z))^{(\alpha+2) / p}} \leq \varepsilon \frac{M}{M_{1}^{(\alpha+2) / p}}
$$

whenever $M_{1} \leq \Im \varphi(z) \leq M_{2}$ and $|\Re \varphi(z)|>M_{3}$.

If $M_{1} \leq \Im \varphi(z) \leq M_{2}$ and $|\mathfrak{R} \varphi(z)| \leq M_{3}$, then there exists some $n_{0} \in \mathbb{N}$ such that $\left|f_{n}(\varphi(z))\right|<\varepsilon$ for all $n \geq n_{0}$, and so

$$
(\Im z)^{\beta}|\psi(z)|\left|f_{n}(\varphi(z))\right|<\varepsilon\|\psi\|_{\mathcal{A}_{\beta}^{\infty}\left(\Pi^{+}\right)} .
$$

Combining (2.27)-(2.32), we have that

$$
\left\|W_{\varphi, \psi} f_{n}\right\|_{\mathcal{A}_{\beta}^{\infty}\left(\Pi^{+}\right)}<\varepsilon C,
$$

for $n \geq n_{0}$ and some $C>0$ independent of $n$. Since $\varepsilon$ is an arbitrary positive number, by Lemma 2.8, it follows that $W_{\varphi, \psi}: \mathcal{A}_{\alpha}^{p}\left(\Pi^{+}\right) \rightarrow \mathcal{A}_{\beta}^{\infty}\left(\Pi^{+}\right)$is compact. 
Example 2.11. Let $1 \leq p<\infty, \alpha>-1$, and $\beta>0$ be such that $\alpha+2=\beta p$. Let $\varphi(z)=z+i$ and $\psi(z)=1 /(z+i)^{\beta}$, then $\Re \varphi(z)=x$ and $\Im \varphi(z)=y+1$. It is easy to see that $\psi \in \mathcal{A}_{\beta}^{\infty}\left(\Pi^{+}\right)$. Beside this, for $z \in \Gamma_{a, b}$, we have

$$
(\Im z)^{\beta}|\psi(z)|=\frac{y^{\beta}}{\left(x^{2}+(y+1)^{2}\right)^{\beta / 2}} \leq \frac{b^{\beta}}{\left(x^{2}+a^{2}\right)^{\beta / 2}} \longrightarrow 0 \quad \text { as } \Re \varphi(z)=x \longrightarrow \infty .
$$

Also

$$
\sup _{z \in \Pi^{+}} \frac{(\Im z)^{\beta}}{(\Im \varphi(z))^{\beta}}|\psi(z)|=\sup _{z=x+i y \in \Pi^{+}} \frac{y^{\beta}}{(y+1)^{\beta}} \frac{1}{\left(x^{2}+(y+1)^{2}\right)^{\beta / 2}} \leq 1<\infty,
$$

and the set $\{z: \Im \varphi(z)<1\}$ is empty. Thus $\varphi$ and $\psi$ satisfy all the assumptions of Theorem 2.10, and so $W_{\varphi, \psi}: \mathscr{A}_{\alpha}^{p}\left(\Pi^{+}\right) \rightarrow \mathcal{A}_{\beta}^{\infty}\left(\Pi^{+}\right)$is compact.

\section{Acknowledgments}

The work is partially supported by the Serbian Ministry of Science, projects III 41025 and III 44006. The work of the second author is a part of the research project sponsored by National Board of Higher Mathematics (NBHM)/DAE, India (Grant no. 48/4/2009/R\&D-II/426).

\section{References}

[1] C. C. Cowen and B. D. MacCluer, Composition operators on spaces of analytic functions, Studies in Advanced Mathematics, CRC Press, Boca Raton, Fla, USA, 1995.

[2] V. Matache, "Composition operators on $H^{p}$ of the upper half-plane," Analele Universităţii din Timişoara, Seria Ştiinţe Matematice, vol. 27, no. 1, pp. 63-66, 1989.

[3] V. Matache, "Composition operators on Hardy spaces of a half-plane," Proceedings of the American Mathematical Society, vol. 127, no. 5, pp. 1483-1491, 1999.

[4] H. J. Schwartz, Composition operators on $\mathrm{H}^{p}$, Ph.D. thesis, University of Toledo, Ohio, USA, 1969.

[5] J. H. Shapiro and W. Smith, "Hardy spaces that support no compact composition operators," Journal of Functional Analysis, vol. 205, no. 1, pp. 62-89, 2003.

[6] A. K. Sharma and S. D. Sharma, "Weighted composition operators between Bergman-type spaces," Communications of the Korean Mathematical Society, vol. 21, no. 3, pp. 465-474, 2006.

[7] S. D. Sharma, A. K. Sharma, and S. Ahmed, "Carleson measures in a vector-valued Bergman space," Journal of Analysis and Applications, vol. 4, no. 1, pp. 65-76, 2006.

[8] S. D. Sharma, A. K. Sharma, and S. Ahmed, "Composition operators between Hardy and Bloch-type spaces of the upper half-plane," Bulletin of the Korean Mathematical Society, vol. 44, no. 3, pp. 475-482, 2007.

[9] S. D. Sharma, A. K. Sharma, and Z. Abbas, "Weighted composition operators on weighted vectorvalued Bergman spaces," Applied Mathematical Sciences, vol. 4, no. 41-44, pp. 2049-2063, 2010.

[10] A. L. Shields and D. L. Williams, "Bonded projections, duality, and multipliers in spaces of analytic functions," Transactions of the American Mathematical Society, vol. 162, pp. 287-302, 1971.

[11] R. K. Singh and S. D. Sharma, "Composition operators on a functional Hilbert space," Bulletin of the Australian Mathematical Society, vol. 20, no. 3, pp. 377-384, 1979.

[12] R. K. Singh and S. D. Sharma, "Noncompact composition operators," Bulletin of the Australian Mathematical Society, vol. 21, no. 1, pp. 125-130, 1980. 
[13] S. Stević, "Weighted composition operators between mixed norm spaces and $H_{\alpha}^{\infty}$ spaces in the unit ball," Journal of Inequalities and Applications, Article ID 28629, 9 pages, 2007.

[14] S. Stević, "Norm of weighted composition operators from Bloch space to $H_{\mu}^{\infty}$ on the unit ball," Ars Combinatoria, vol. 88, pp. 125-127, 2008.

[15] S. Stević, "Weighted composition operators from weighted Bergman spaces to weighted-type spaces on the unit ball," Applied Mathematics and Computation, vol. 212, no. 2, pp. 499-504, 2009.

[16] S. Stević, "Composition operators from the Hardy space to Zygmund-type spaces on the upper halfplane and the unit disc," Journal of Computational Analysis and Applications, vol. 12, no. 2, pp. 305-312, 2010.

[17] S. Stević, "Composition operators from the Hardy space to the $n$-th weighted-type space on the unit disk and the half-plane," Applied Mathematics and Computation, vol. 215, no. 11, pp. 3950-3955, 2010.

[18] S. Stević, "Composition operators from the weighted Bergman space to the $n$-th weighted-type space on the upper half-plane," Applied Mathematics and Computation, vol. 217, no. 7, pp. 3379-3384, 2010.

[19] S.-I. Ueki, "Hilbert-Schmidt weighted composition operator on the Fock space," International Journal of Mathematical Analysis, vol. 1, no. 13-16, pp. 769-774, 2007.

[20] S.-I. Ueki, "Weighted composition operators on the Bargmann-Fock space," International Journal of Modern Mathematics, vol. 3, no. 3, pp. 231-243, 2008.

[21] S.-I. Ueki, "Weighted composition operators on some function spaces of entire functions," Bulletin of the Belgian Mathematical Society Simon Stevin, vol. 17, no. 2, pp. 343-353, 2010.

[22] X. Zhu, "Weighted composition operators from area Nevanlinna spaces into Bloch spaces," Applied Mathematics and Computation, vol. 215, no. 12, pp. 4340-4346, 2010.

[23] F. Forelli, "The isometries of $H^{p}$ spaces," Canadian Journal of Mathematics, vol. 16, pp. 721-728, 1964.

[24] C. J. Kolaski, "Isometries of weighted Bergman spaces," Canadian Journal of Mathematics, vol. 34, no. 4, pp. 910-915, 1982.

[25] S. Li and S. Stević, "Generalized composition operators on Zygmund spaces and Bloch type spaces," Journal of Mathematical Analysis and Applications, vol. 338, no. 2, pp. 1282-1295, 2008.

[26] S. Stević, "On a new operator from $H^{\infty}$ to the Bloch-type space on the unit ball," Utilitas Mathematica, vol. 77, pp. 257-263, 2008.

[27] S. Stević, "On an integral operator from the Zygmund space to the Bloch-type space on the unit ball," Glasgow Mathematical Journal, vol. 51, no. 2, pp. 275-287, 2009.

[28] S. Stević, "On a new integral-type operator from the Bloch space to Bloch-type spaces on the unit ball," Journal of Mathematical Analysis and Applications, vol. 354, no. 2, pp. 426-434, 2009.

[29] S. Stević, "On an integral operator between Bloch-type spaces on the unit ball," Bulletin des Sciences Mathématiques, vol. 134, no. 4, pp. 329-339, 2010.

[30] X. Zhu, "Generalized weighted composition operators from Bloch type spaces to weighted Bergman spaces," Indian Journal of Mathematics, vol. 49, no. 2, pp. 139-150, 2007.

[31] K. L. Avetisyan, "Integral representations in general weighted Bergman spaces," Complex Variables, vol. 50, no. 15, pp. 1151-1161, 2005.

[32] K. L. Avetisyan, "Hardy-Bloch type spaces and lacunary series on the polydisk," Glasgow Mathematical Journal, vol. 49, no. 2, pp. 345-356, 2007.

[33] K. D. Bierstedt and W. H. Summers, "Biduals of weighted Banach spaces of analytic functions," Australian Mathematical Society Journal Series A, vol. 54, no. 1, pp. 70-79, 1993. 


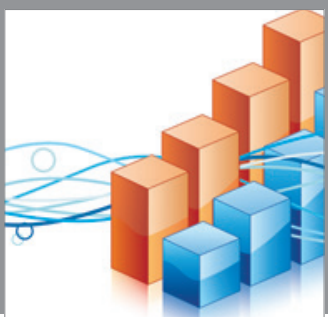

Advances in

Operations Research

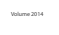

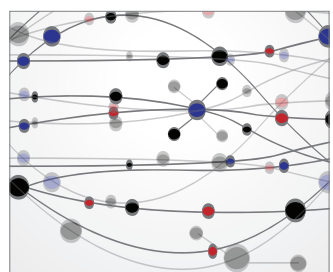

\section{The Scientific} World Journal
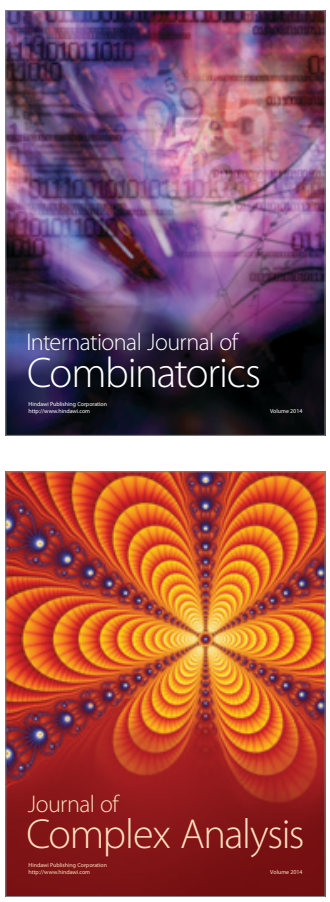

International Journal of

Mathematics and

Mathematical

Sciences
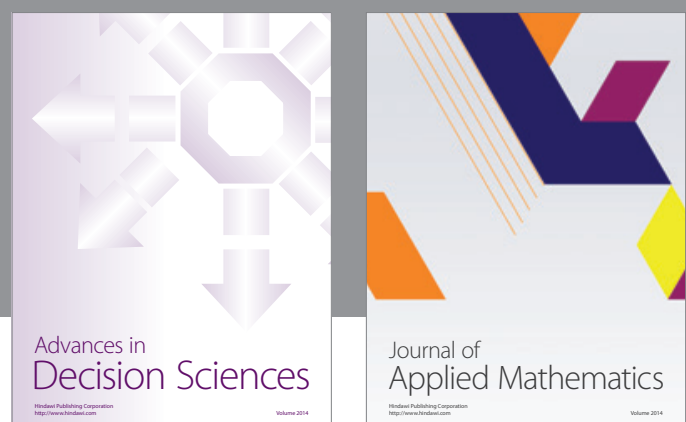

Journal of

Applied Mathematics
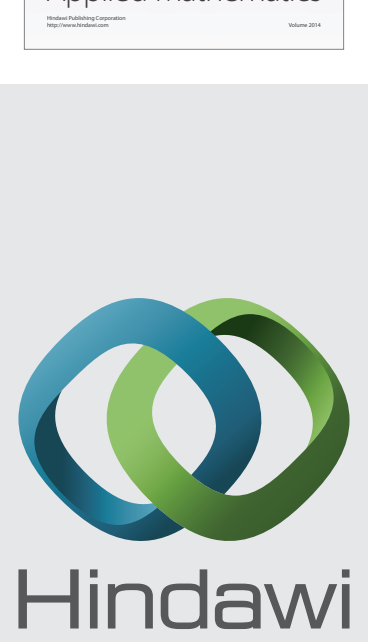

Submit your manuscripts at http://www.hindawi.com
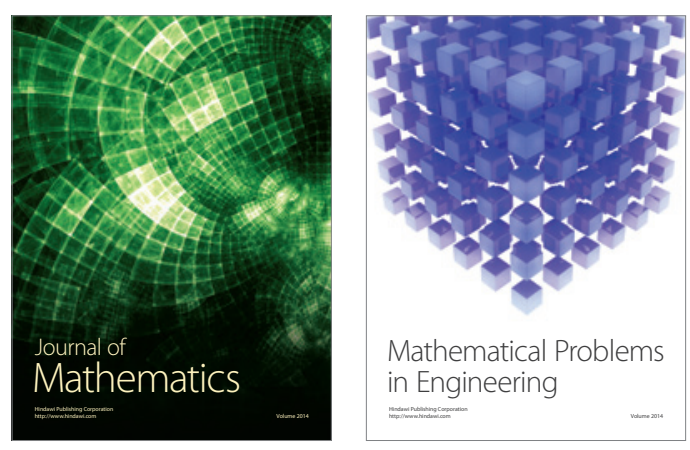

Mathematical Problems in Engineering
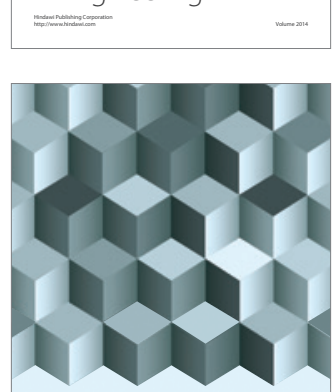

Journal of

Function Spaces
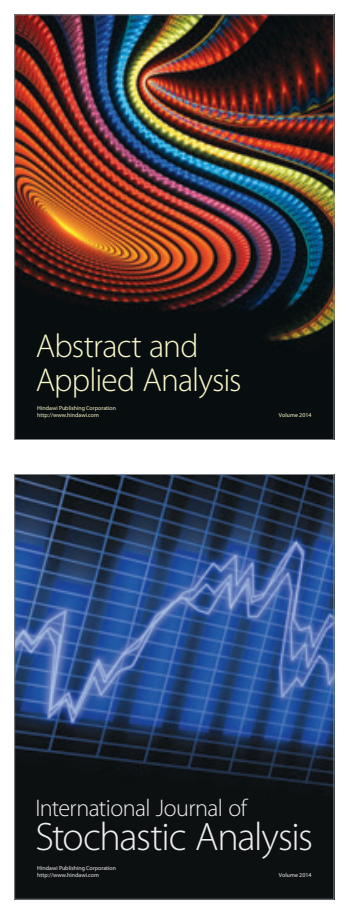

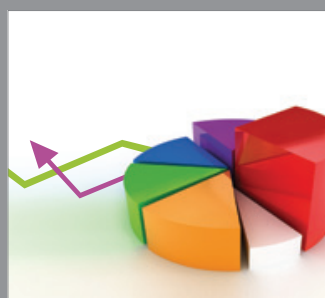

ournal of

Probability and Statistics

Promensencen
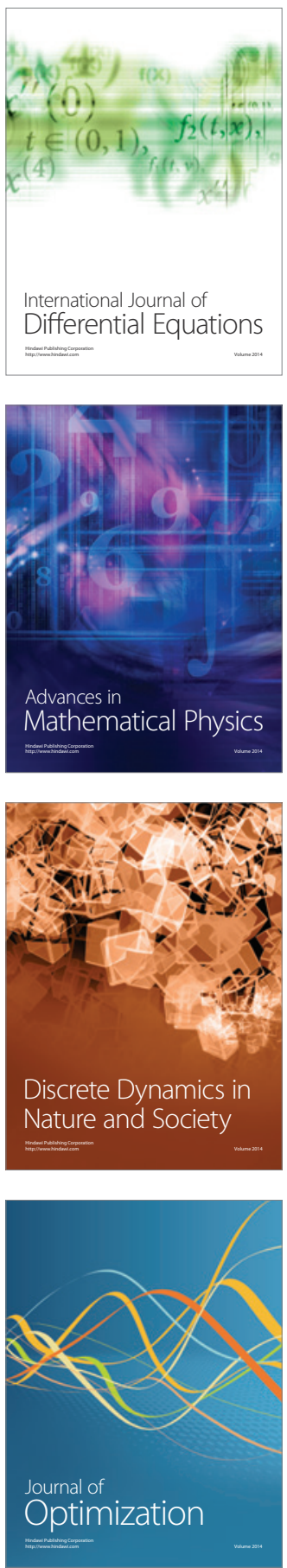